\title{
Designing the Virtual River Game to support the collaborative exploration of river interventions
}

Robert-Jan den Haan, Mascha van der Voort, Fedor Baart, Koen Berends \& Suzanne Hulscher

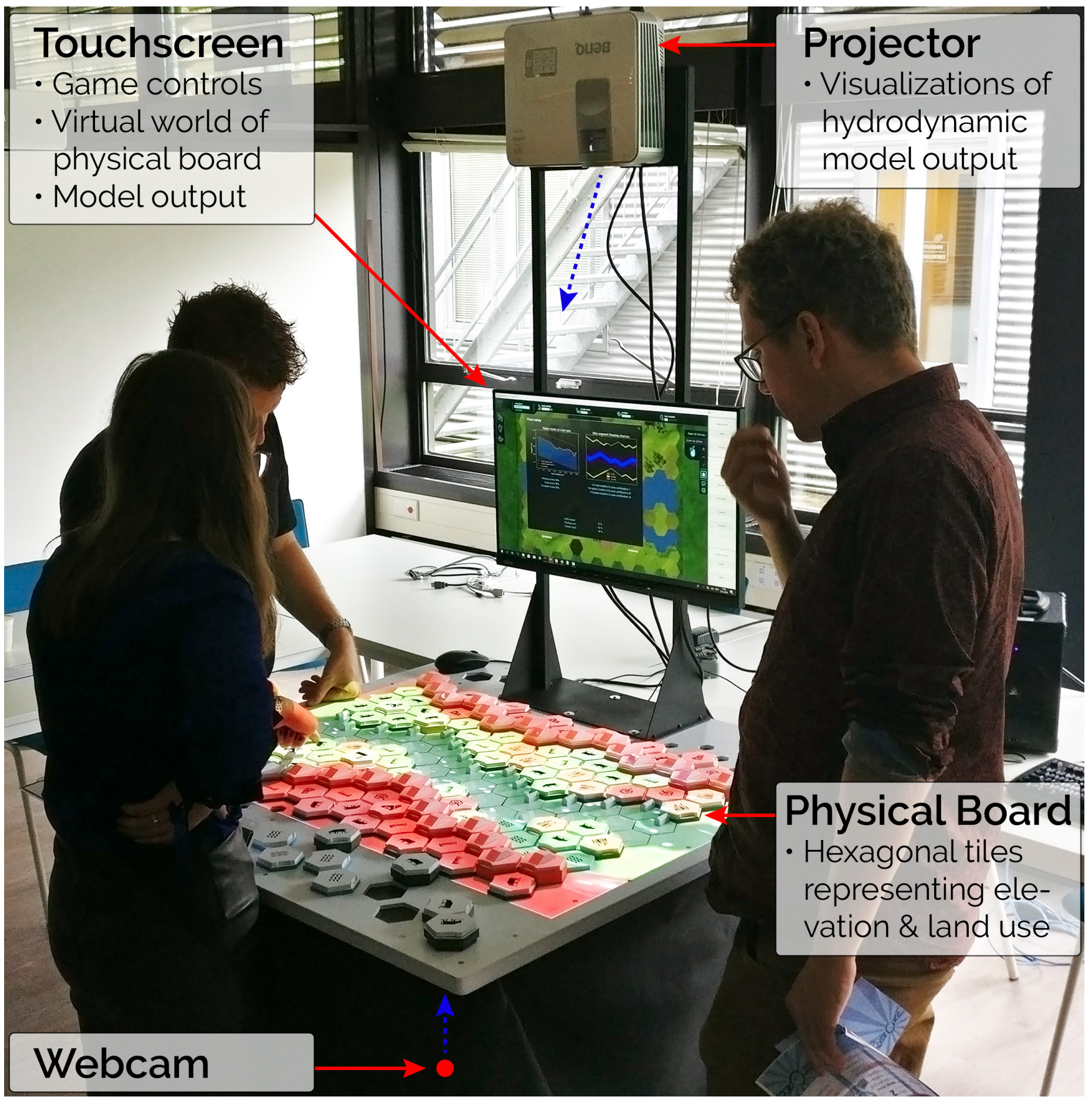

An impression of the Virtual River Game's setup, including the physical board, touchscreen monitor, projector, and webcam. Each hexagon tole on the board is filled with modular game pieces that define the tile's elevation and land use. In combination, the board represents a fictional, but typical, stretch of a Dutch river. The board is directly connected to a $2 \mathrm{D}$ hydrodynamic model to compute the hydraulic effects of changes on the board. The projection on the board in this figure shows the hydraulic roughness of floodplain and channel locations, visualized in a green to red color range to represent smooth to rough. The touchscreen monitor has two functions: (1) as a controller for players to initiate model updates and to switch visualizations; and (2) as an overview of the current state in the game by providing a virtual world of the board as well as information on the in-game performance (Den Haan et al., 2020). 

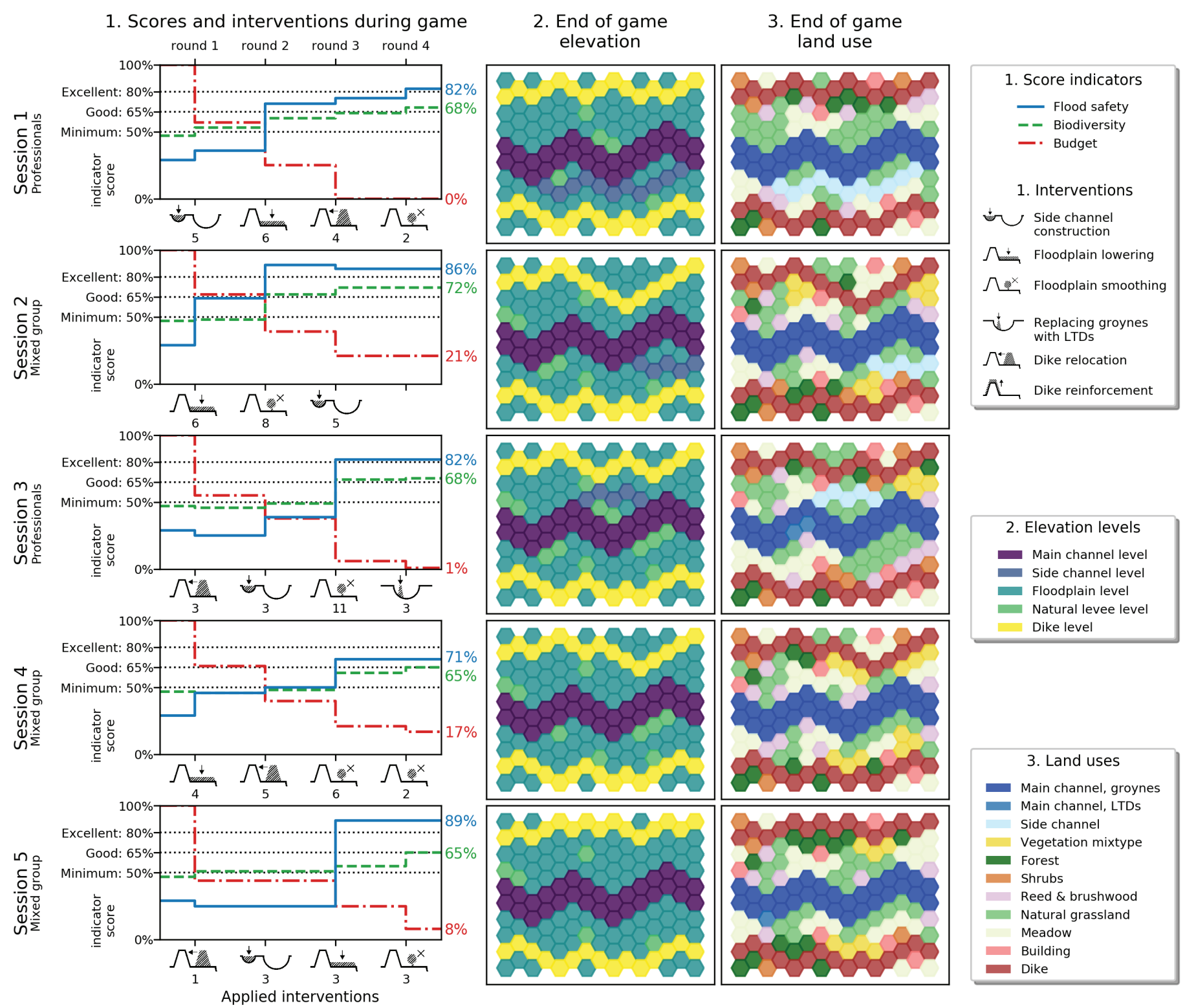

2. Elevation levels

Main channel level

Side channel level

Floodplain level

Natural levee level

Dike level

3. Land uses
Main channel, groynes
Main channel, LTDs
Side channel
Vegetation mixtype
Forest
Shrubs
Reed \& brushwood
Natural grassland
Meadow
Building
Dike

Overview of the players' in-game actions of five game sessions, with each row showing the result of one session. The first column shows the interventions applied during the four game rounds and the resulting progression of indicator scores, with the final indicator scores listed to the right of the graphs. The number shown below each intervention icon indicates the number of implementations that participants tested during the respective round. The budget indicator scores for the first two sessions are adjusted to match the lowered budget. The second column shows the final board layout for the bed elevation and the third column shows the final layout for the land use (Den Haan et al., 2020). 


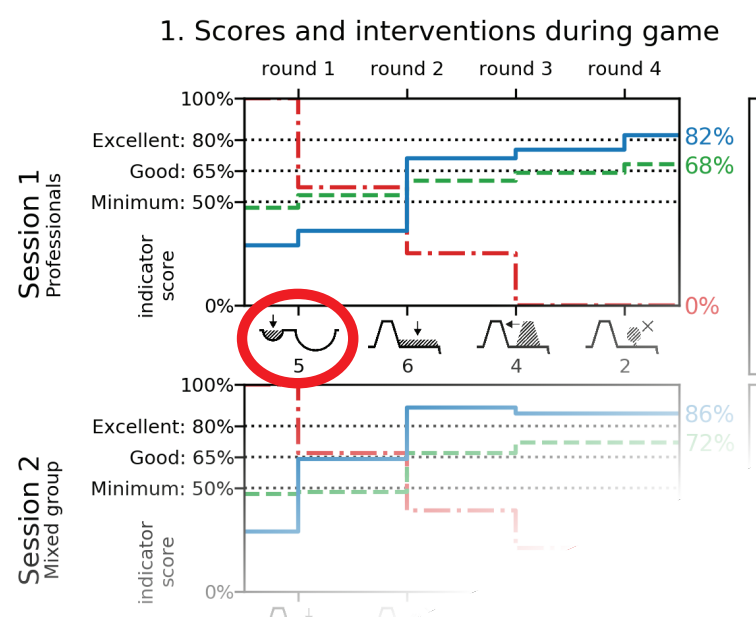

2. End of game $\quad 3$. End of game land use

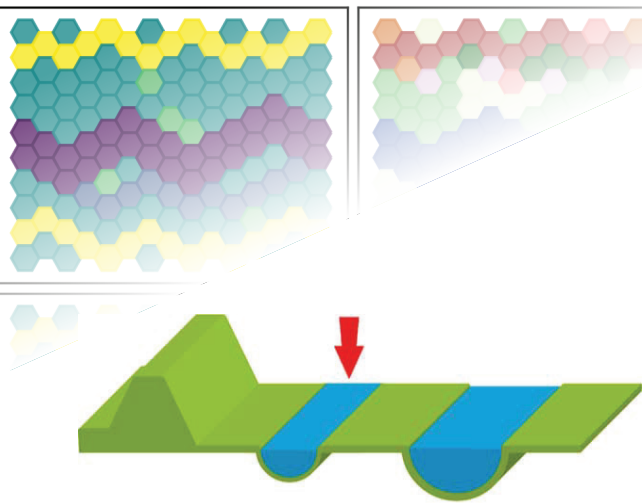

Side channel construction

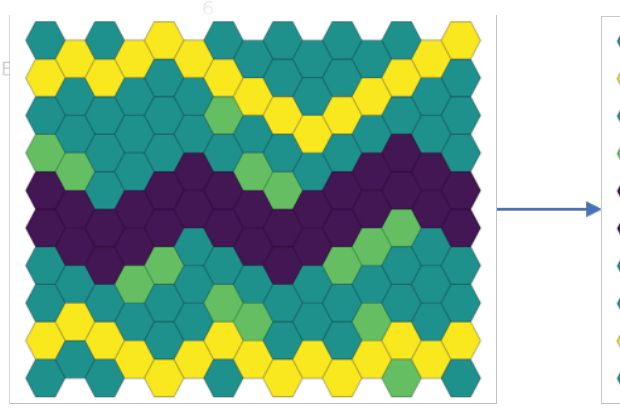

0

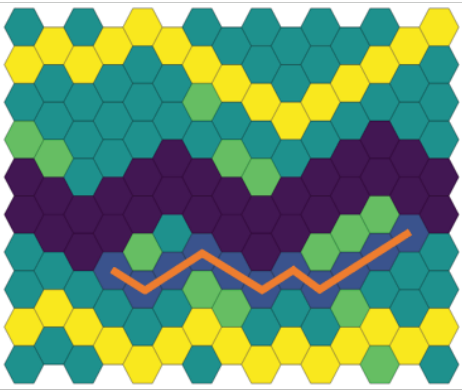

1

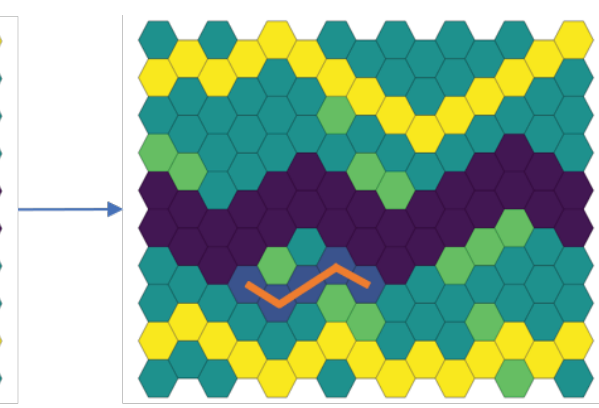

2

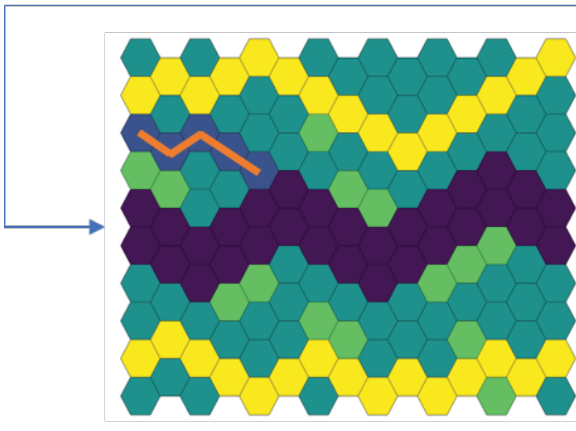

3

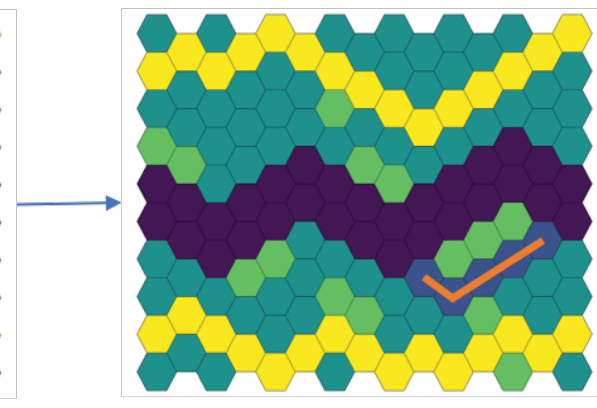

4

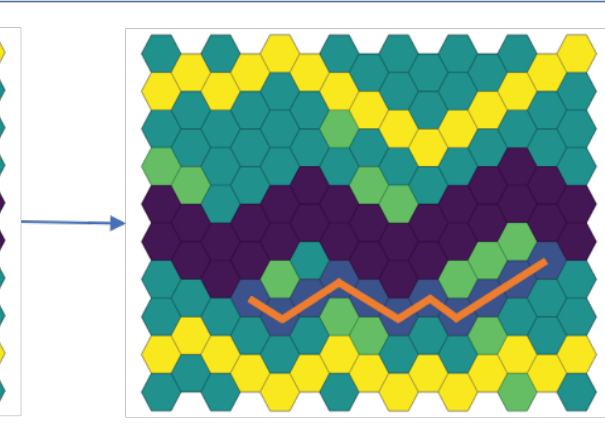

5

Example of the experimentations that players did while playing the game. In the first turn of the first session, players applied and tested five implementations of a side (or secondary) channel construction. To do so, players placed lower game pieces in the floodplain, resembling an excavation, in such a way that the created channel connects to the main channel on at least one side. The game's hydrodynamic, ecological and cost model computes the effect of each applied intervention. The players subsequently discuss the effects of the applied intervention and whether or not they wish the test another implementation. The game round ends when players agree with making an implementation final. 

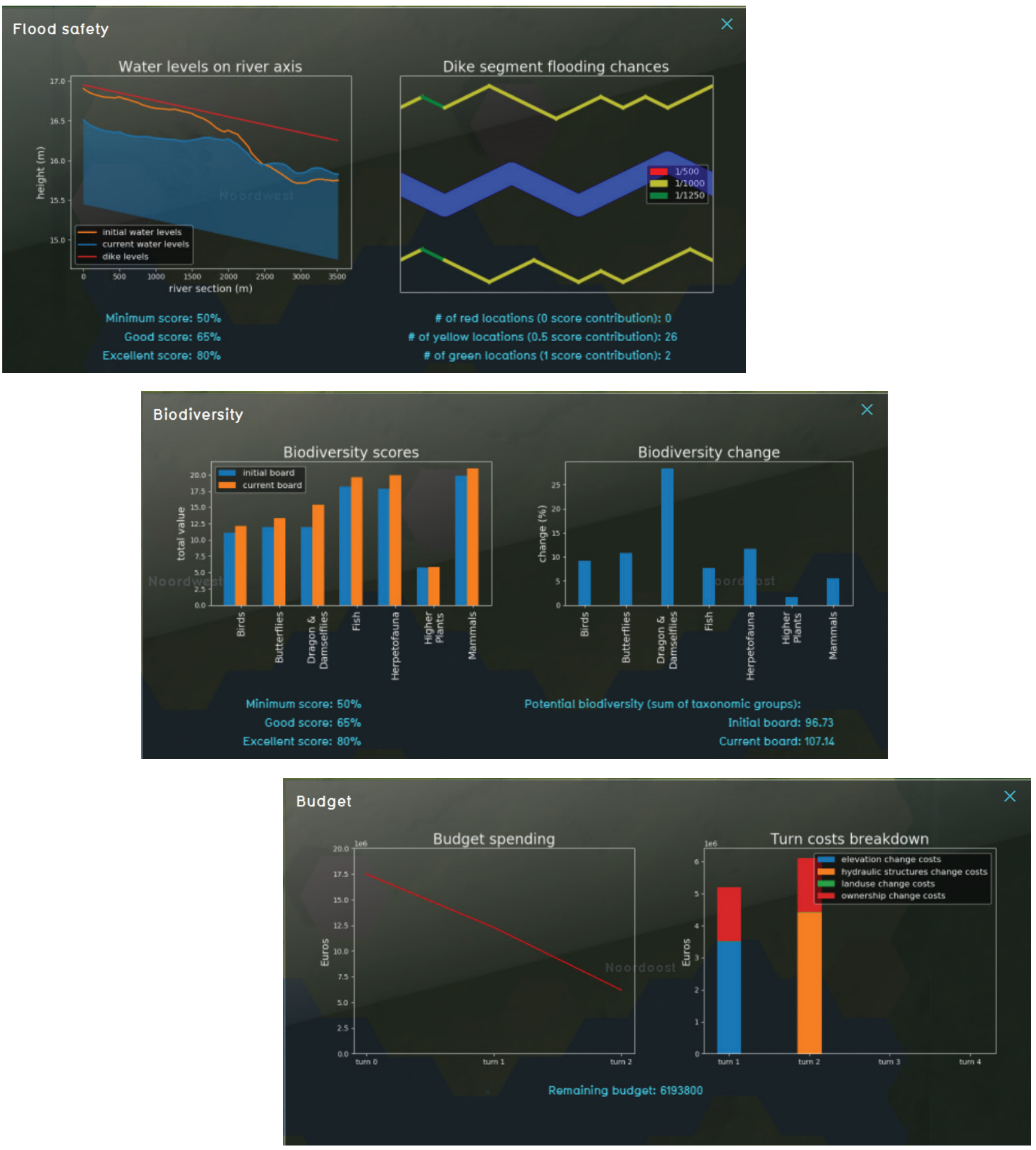

In-game feedback on the effects of interventions regarding flood safety (top), biodversity (middle) and budget (bottom), corresponding to the integrated hydrodynamic, ecological and cost models. This feedback is available to players on the touchscreen monitor, in the Tygron Geodesign Platform that shows the virtual world of the game area. 
Participant self-reported overall impressions

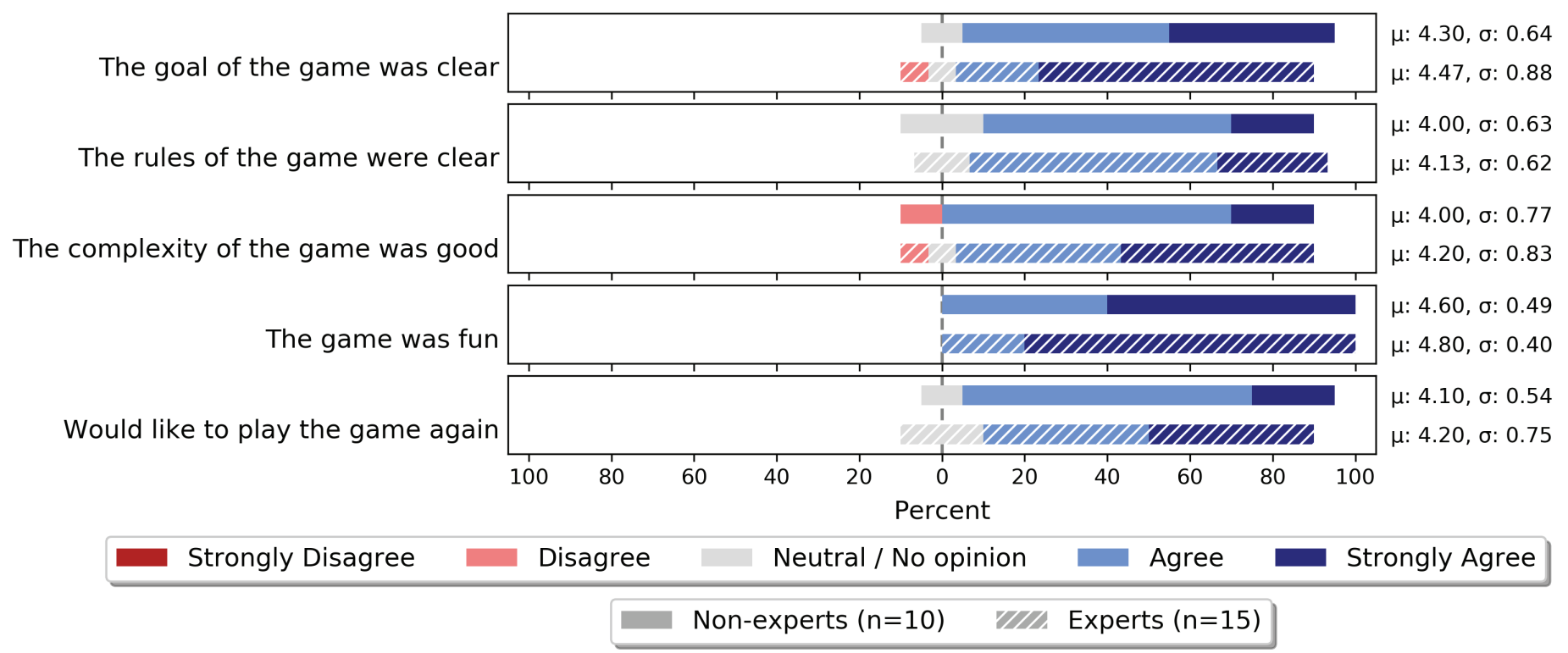

Overall impressions of the game as reported in the post-game questionnaire based on a 5-point Likert scale. Rating strongly agree counts as 5; strongly disagree as 1 (Den Haan et al., 2020) 
Participant self-reported insights

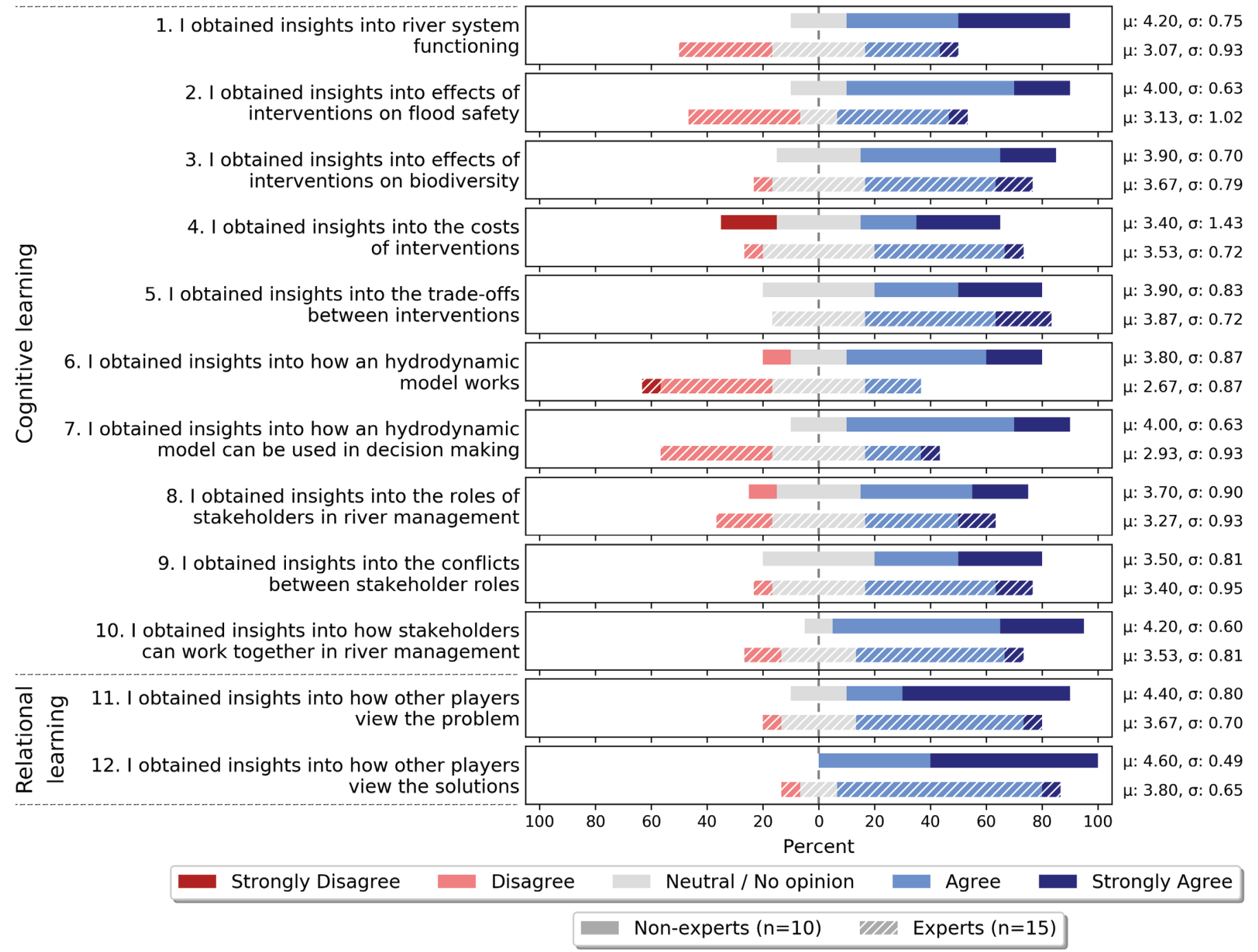

Learning outcomes of the game as reported in the post-game questionnaire based on a 5-point Likert scale. Rating strongly agree counts as 5; strongly disagree as 1 . The statements relate to the functioning of the river system (1), the effects of interventions and their trade-offs $(2-5)$, how hydrodynamic models work and are used in decision-making (6-7), the roles of and conflicts between stakeholder roles (8-10), and the views and perspectives of other players (11-12) (Den Haan et al., 2020)

More information:

Den Haan, R., Van Der Voort, M., Baart, F., Berends, K. D., Van Den Berg, M., Straatsma, M. W., ... \& Hulscher, S. J. M. H. (2020). The Virtual River Game: Gaming using models to collaboratively explore river management complexity. Environmental Modelling \& Software, 134, 104855. 